\title{
The Territory Is the Map: Exploring the Use of Landmarks in Situ to Inform Mobile Guide Design
}

\author{
Nicola J. Bidwell ${ }^{1}$ and Jeff Axup ${ }^{2}$ \\ ${ }^{1}$ School of IT, James Cook University, Cairns, Queensland, Australia 4878 \\ nic@it.jcu.edu.au \\ ${ }^{2}$ School of IT and Electrical Engineering, University of Queensland, Australia 4172
}

\begin{abstract}
People have difficulties interacting with external representations designed to guide navigating physical environments. We derive theory to inform design by probing users' experience and use of their "internal" representations in a temporally evolving wayfinding activity in situ. Interactions with environmental landmarks are explored by analyzing spatial concepts in SMSs used by a group collaborating to wayfind to an unfamiliar rendezvous. Results show differences between landmarks provoking actions and contributing to abstract concepts; and, effects of direct or induced perspective in situ. Design recommendations account for orientation dependence and use of ambiguity in user-world-representation mappings. These include tactics to enable users' to induce perspectives appropriately: with accuracy for recognising landmarks along routes and agility to situate landmark use in naturally evolving wayfinding goals.
\end{abstract}

\section{Introduction}

People encounter difficulties interacting with external representations designed to guide wayfinding physical environments [e.g. 1, 2]. Here, we derive theory to inform design by probing users' experience and use of their internal representations in situ. First, we note close couplings between wayfinding and more global goals and the importance of landmarks in mappings between people and terrain. Then we describe an approach exploring mappings in "naturally" evolving interactions in situ. This analyses spatial concepts in Short Message Service (SMS) communicated and used by a group collaborating to wayfind to an unfamiliar rendezvous. We summarise the method and a narrative of the activity. Next, we describe generalities of landmarks use and themes abstracted by grouping observations related to perspective and different wayfinding goals. This illustrates the effect of orientation and situated goals on mappings and their relations with users' directly experienced or induced perspective. We conclude with design recommendations to assist users in reconciling their internal representations and experience in situ and relate these to situated and collaborative wayfinding. We propose specific tactics to guide perspective enabling landmark recognition en route and to confer agility in using landmarks for naturally evolving wayfinding goals.

\subsection{Landmarks in Mappings Between People and the World While Wayfinding}

Wayfinding encompasses going to both familiar and unfamiliar destinations and exploring unfamiliar terrain. While wayfinding may be implicit to all mobile activity it 
is rarely the goal. Instead, it is an activity which is closely coupled with the person and their environment and more global goals (e.g. go to work, meet friends, sightsee). A person's interactions while fluidly traversing familiar terrain are "thrown" [e.g. 3]. This implies wayfinding perceptions and actions in such, "ready-to-hand", terrain are unreflective and peripheral to other goals, perceptions and actions. Reliable "thrown" interactions depend on effective mappings [4] between the environment represented internally and experienced in situ. In unfamiliar terrain people interact more reflectively and focus on wayfinding perceptions and actions. Mappings with the environment in situ also reflect dynamic and subjective contextualisation [e.g. 5] of internal representations by perceptions, conceptions and actions associated with people's global goals. Consider a pair of tourists agreeing to meet for ice-cream and explore Australia's famous Bondi Beach for the first time. For example, either's internal representation may be contextualised by semantics conferred to gelateries; physical relations between gelateries, clusters of people and promenades; and, temporality and intersubjectivity such as anticipating their, and the other person's, time and direction of arrival etc.

Landmarks are locational cues that link internal representations to perceptions and actions in the physical environment. The conception of space as sets of familiar landmarks has been shown cognitively, behaviourally and phenomenologically $[6,7,8]$. Landmarks are salient to acquiring, communicating and applying spatial knowledge in wayfinding. People use landmarks to learn [9], use and describe [10] routes between places and for higher-level cognitive concepts allied with wayfinding (e.g. selecting an area in which to dine or shop [11]). Landmarks in route guidance are most effective when they are proximal to a route $[12,13]$ and relate to prescribed actions [14]. However, meta- and structural information in people's route descriptions [2] seems to express an appreciation of the recipient's global navigation goal [15]. This may contribute to internal representations of landmarks which differ from route sequences.

External representations designed to support wayfinding in mobile devices explicitly or implicitly integrate landmarks [e.g. 16]. Landmarks act as decision points and/or spatial references in route descriptions of different modalities, graphical depictions, (e.g. annotated maps) and, photographic or simulated, transcendent or immanent views. Interaction difficulties suggest external representations of landmarks may not map closely to users' internal representations and experience in situ. This may relate to the salience of landmarks for effective wayfinding. Landmark salience includes its visual characteristics, semantics (e.g. purpose) and its relative location in the structure of the environment [17]. Differences arise between the types of landmarks described from memory and during route creation in situ [18]. Since external representations tend to be designed outside of an environment they favour a landmark's salience for recall and particular semantic concepts associated with places and routes. This may not map well to a user's internal representation and immanent experience of landmarks along unfamiliar routes. Evidence identifying the effect of perspective on internally representing objects [19] suggests mappings are prone to conflicts between the perspectives of users in situ and those of external representations. In unfamiliar terrain, users have difficulties or make errors when matching their perspective with immanent 2-D views [13, 20], and dynamically transposing [1] or self-reporting their position [21] with extrinsic depictions (e.g. aerial-view maps). Further, salience may be subjective [17], which may account for differences noted between users [20] and is likely to temporally evolve with global or situated goals. People refer to external representations of landmarks during situated, as 
well planned, wayfinding; for example, tourists use landmarks in situ as loci to pivot exploring interesting areas [11].

\section{Interactions During an Evolving Wayfinding Activity}

\subsection{Probing Users' Experience and Use of Internal Representations in Situ}

To develop insight into people's mappings during wayfinding we drew upon two interaction design approaches: phenomenological games and technology probes. We sought to provoke foregrounded perceptions and actions in the environment by framing wayfinding as a spatially-authentic, mobile, collaborative problem solving experience. This contrasts with studies which foreground activity around an external representation by accounting for users' interactions with a system (e.g. a mobile guide) and the world [e.g. $11,20]$. A group of "wayfinders", starting from separate undisclosed locations, searched for and rendezvoused at an unfamiliar target, a small park (Fig. 4a). They were guided only by a brief description of the target (Fig. 1b) and their interactions with the physical environment and each other. We used mobile SMS to capture data on spatial concepts associated with wayfinding during the activity. Wayfinders communicated with each other using SMS only, even if they were in sight of each other. Every SMS was broadcast to the other wayfinders and could be referred to repeatedly. Ethnographic evidence suggests people often collaborate to wayfind [11], use SMS to rendezvous [22] and evolve plans amongst groups from numerous short messages [23]. In logging each SMS the device acted as a technology probe, by possessing "embedded, invisible, nonintrusive functionality enabling ongoing use of the technology" [24]. In addition to formative, holistic understandings, as commonly yielded by probes, we also systematically analysed the situated use of spatial concepts to inform interpreting mappings. We posited that SMS content would verbally describe conceptual categories and linguistic structures [in: 17] associated with wayfinders' perceptions and actions in the environment and temporally evolving activity. Thus, SMSs may embody some "thrown" interactions and situated and global goals. Length constraints on SMS restrict verbosity. To inform interpreting indexicality [25] we captured detailed in situ observations and spatio-temporal and post-activity reflective data.

\subsection{Method}

Mobile activity covered an $8 \mathrm{~km}^{2}$ extent in Palmerston, Northern Territory, Australia. This included parts of the Central Business District (CBD) and two proximal suburbs, comprising single-story family homes with gardens. The city, purpose-built in 1981, has a small population, is set in bushland and was planned to account for motor traffic [26]. Wayfinders, referred to as $\mathcal{A}, \mathcal{B}$ and $\mathcal{C}$, were males in their 20 s who volunteered, as a group, via a WiFi gaming community website. All had been to Palmerston but only $\mathcal{C}$ knew parts of the suburbs. $\mathcal{B}$ and $\mathcal{C}$ had walked around the $\mathrm{CBD}$ but $\mathcal{A}$ had only driven "across some of these main junctions".

The SMS interface to a Nokia 3650 mobile phone was customized as a discussion list between the 3 wayfinders (Fig. 1a). Each wayfinder's SMS was sent to an address book item labelled 'Mobile group'. This was the number of a phone connected, via Bluetooth, to an SMS server running on a laptop in a car parked in the CBD. The 
SMS server [27] recorded the message text, sender and time of arrival of incoming SMSs. It then prefixed "Member $[\mathcal{A}, \mathcal{B}$, or $\mathcal{C}]$ says:" to the SMS and sent it, via the attached phone, to the other two wayfinders without returning a copy to the sender.

Wayfinders and the research team drove to the main shopping centre in the CBD via main roads at least $1.5 \mathrm{Km}$ from the target. Wayfinders were driven individually to separate locations where they waited until an SMS indicated the start of the activity. $\mathcal{A}, \mathcal{B}, \mathcal{C}$ started from the University bus-stop, central bus terminus and in the suburb of Grey respectively (Fig. 2a). Wayfinders were observed passively and occasionally expressed their thoughts and intentions using the Think Aloud protocol. Observers took time-stamped notes and photographs. Each of the 184 photos showed the wayfinder's orientation and what they might be viewing when they received or composed SMSs, or appeared to examine the terrain; or, other notable objects. The next day a pair of observers retraced each wayfinder's route using the notes and photos rendered on a Hewlett Packard Tablet computer (Fig. 1c). They plotted, on a map, each photo's exact position and orientation. Later this data, indexed with times and SMS sent and received, was transposed to an aerial photo from 1,000m (Fig. 2a).

Pre- and post- activity briefings and interviews took place $20 \mathrm{~km}$ east of Palmerston and were recorded on paper and audio. Before the activity, wayfinders were briefed and practiced using the SMS interface. Experiences relevant to the activity were collected by interview and questionnaire. In a post-activity workshop wayfinders scrolled through the SMSs on their phones and photographs of their surroundings at the time were projected onto a wall. Wayfinder's read out each SMS and commented on their actions and experiences related to this. After individual journey reflections, the group discussed confluences and conflicts in their narratives, decisions and SMSs. The reflections prompted specific questions on individual situated perspectives, shared location awareness and usage issues for mobile, group communication.

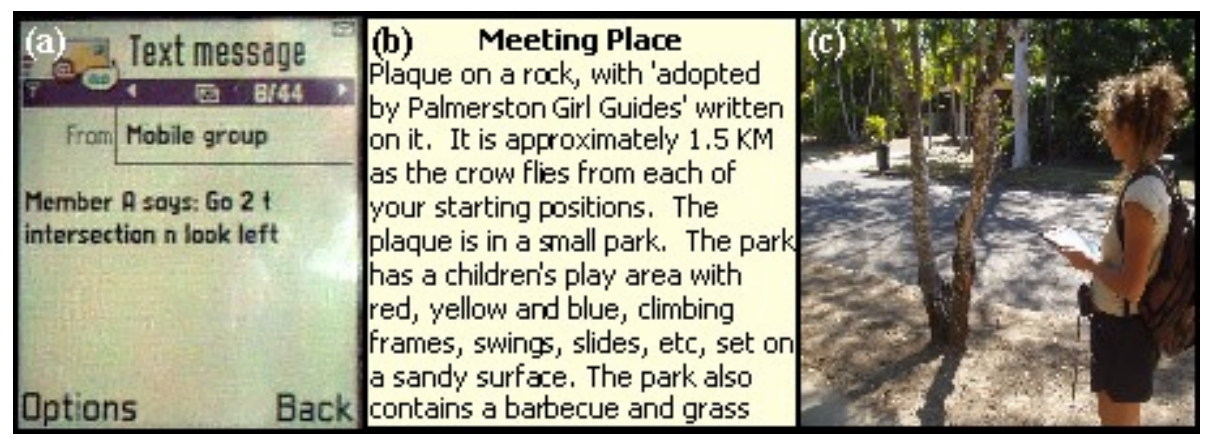

Fig. 1. (a) Mobile phone interface to the group SMS; (b) Description of the target destination; (c) Observer retracing a wayfinder's route using photos rendered on a Tablet PC

\subsection{Results and Interpretations}

Results, first emerging phenomenally, were analysed by integrating the diverse data set. A comprehensive account, incorporating the multiple, distributed data sources, enabled hermeneutically interpreting subtleties of situations. Detailed analysis of the SMS text related to wayfinder's goals, situations and experiences emerging, spatio- 
temporally, during the activity. The first author's analysis was verified by the second author. Here, we emphasise analysis of the use of landmarks and Frame of Reference (FOR) in SMS. A FOR is any co-ordinate system which spatially relates objects and components of objects. It is specified by its origin, orientation and the relationship between its axes. FORs varied with the sender's situation and experience in the environment. Several themes, arising from grouping analyses of landmarks' FORs and indexicality, afford insight into mappings between wayfinders' internal representations and the world. These are discussed (2.4), with example situations according to the use and efficacy of different perspectives of landmarks for various aspects of wayfinding.

\section{Goals of the Wayfinding Activity}

All wayfinders reached the target within 1.5 hours after the first SMS. Their situated wayfinding activity evolved around a set of goals which emerged "naturally" in 5 chronological phases: localize others; primed rendezvous; naïve search for the target; localize target; and, route to target (Figs. 2b,c,d).

Localise Others and Primed Rendezvous: In the first few minutes the majority of SMSs referred to what wayfinders could see, or knew about, their respective starting locations (Fig. 2b). The remainder related to a strategy to deduce the target's location by triangulation. Wayfinders spent time scanning the vicinity of locations. Then each undertook wayfinding which we refer to as "primed" since the all headed to the unanimously familiar water tower (Fig. 2b).

Naïve Search for the Target: From the water tower wayfinders dispersed until $\mathcal{A}$ discovered the target (Fig. 2c). $\mathcal{A}$ walked north, $\mathcal{B}$ walked west and $\mathcal{C}$ south-east. Each headed towards different areas that they already knew existed. They intended to check promising areas or to explore unknown areas. Wayfinders "naïvely" searched for the target as they had no direct experience of either its location or appearance. $\mathcal{A}$ and $\mathcal{B}$ also explored areas naively, commenting "Where the hell [sic] am I?" When $\mathcal{A}$ found the target, $\mathcal{C}$, who was some distance away, asked if it was in the suburb of Driver. Both $\mathcal{A}$ and $\mathcal{B}$ had been in Driver for the past 30 minutes, but neither knew its name. Locating the target was difficult for wayfinders. $\mathcal{A}$ said "The hard part is working out the end point" and $\mathcal{B}$ felt as if he was "aimless[ly] wandering". As, a group, wayfinders maintained distance between each by drawing on having seen each other (e.g. near the water tower $\mathcal{A}$ saw $\mathcal{B}$ head towards a park so did not check it) or tracking each other using SMS information combined with existing area knowledge. Individual strategies differed according to wayfinder's familiarity with Palmerston. $\mathcal{A}$ paused little and operated "on the principle of moving as quickly as possible". This corresponds with people in unfamiliar terrain who modify hastily constructed routes, opportunistically $[11,28]$. Possibly, $C$ moved most slowly as he had better familiarity and sought to limit his time en route by a priori route creation [28].

Localise Target and Route to Target: $\mathcal{A}$ found the target 50 minutes after the first SMS was sent (Figs. 4a, f, g). In the final 37 minutes $\mathcal{A}$ attempted to guide $\mathcal{B}$ and $\mathcal{C}$ to the target (Fig. 2d). He started by describing the target's location but by the end included specific route guidance. 


\section{Landmarks Referred to in SMSs}

The majority $(83 \%)$ of the 56 SMSs exchanged were reports, questions or instructions relating to location and/or direction. Of these $72 \%$ referred to one or more landmarks and the remainder contained non-specific locational and/or directional information (e.g. "where to now?"). Current or intended self movement was specified in 12 reports relating a landmark to verbs (e.g. search, turn, pass). There were 9 instructions for a direction of heading by another wayfinder which related a landmark to the verbs: head, check, go and look. Throughout wayfinding SMSs referred to landmarks that the sender both was or was not currently able to see. Of all instances of landmarks described in SMSs the sender was able to see or had very recently seen, $43 \%$; or had previously seen $37 \%$ which were not currently visible. The remaining $19 \%$ had not yet been seen by the sender during the activity. Only $14 \%$ of landmarks mentioned were never seen by the sender during the activity. The frequency of referring to a landmark did not correlate with time spent in its vicinity (e.g. wayfinders walked on University Avenue across the longest duration but referred to it only at the start and end).

Landmarks were used to refer to a location in $60 \%$ of all SMSs. The landmarks can be classified according to Lynch's schema [7]. The most frequent class was a "node", where people undertake activities. Eight nodes were visible, and one was signposted, from main arterial roads. The 3 nodes that were not visible or sign posted from main arterial roads required fuller description by relating to other landmarks. SMSs mentioned 8 "districts", or larger entities with particular semantic identities [7]: 6 visible from main arterial roads and, 2 signposted from main arterial roads. All of the 6 "edges" indicated in SMSs had fuller descriptions by relating to other landmarks; 4 were road features and 2 were distinctly labelled entrances. All 4 "paths" were road names. There was one Lynchian "landmark", the water tower.

Approximately half $(56 \%)$ of SMSs which used landmarks to describe a location expressed directional information. A total of 29 different landmarks were each specified between 1 and 10 times (mean $=3$ per landmark). Landmarks were described by name or function in equal proportion. Those described by name included districts such as suburbs (e.g. "Driver"); paths, such as roads (e.g. "University Avenue"); or nodes such as shops (e.g. "Woolworths"). Name usage closely related to functional uniqueness (e.g. 3 supermarkets were individually named). Landmarks described by function included areas (e.g. "golf course"), buildings (e.g. "shopping centre"), distinct features (e.g. "water tower") or road features (e.g. roundabout) (Figs. 2b,c,d). Parts of landmarks described specifically were considered distinct (e.g. $13^{\text {th }}$ hole [of golf course]; Corner of [Chung Wah Terrace]). SMSs frequently described locational and/or directional information by combining several landmarks which are considered distinct. For example, "Oasis-Target roundabout" contains: Oasis, Target and roundabout. Composite descriptions were used to provide specific locational descriptions and/or directional information. Some SMSs described two locations to provide directional information. For example, "Circling Coles to water tower" refers to a direction of movement towards another location. Such a SMS is considered to contain one directional and two locational information items. Some SMSs contained several locational and/or directional information items. For example," Check the skate park then head towards fairway waters, $\mathcal{B}$ check near golfpark I'll go down main middle 
road" contains 4 locational items (skate park, golf park, fairway waters and main middle road) and one explicit directional information item: "then head towards".

\section{Perspectives of Frames of Reference (FORs)}

SMSs tended to refer to landmarks with an explicit FOR (total $=54$ information items). The majority of SMSs explicitly referred to landmarks with intrinsic FORs, with co-ordinates within the world (e.g. Table 1). Many information items (48\%) with explicit FORs related landmarks to the sender's own, egocentric, perspective (e.g. "The first left before the golf course"). Other information items (33\%) with explicit FORs described the landmark, allocentrically from the landmark's perspective (e.g. "Inside Fairway Waters"). Many intrinsic FORs were induced [29] indirectly as the sender was not insight of the landmark at the time. Wayfinders must have induced at least $44 \%$ of egocentric and 32\% of allocentric FORs in SMS as they referred to landmarks that the sender had not yet seen in the activity. One SMS used extrinsic FOR with co-ordinates lying outside of the world and induced from experience (Table 1). However, wayfinders were uncertain in relating locations to absolute directions.

Table 1. Examples of SMS (referred to in text), landmarks and FORS

\begin{tabular}{|l|l|l|l|}
\hline \multicolumn{1}{|c|}{ SMS Text } & \multicolumn{1}{c|}{ Landmarks } & \multicolumn{2}{c|}{ FOR } \\
\hline omw [on my way] & \multicolumn{2}{c|}{-} & Egocentric \\
\hline Circling coles 2 tower & Coles; Watertower & Allocentric & Direct \\
\hline Bhind the shopin centre target & Shopping centre; Target & Allocentric & \\
\cline { 1 - 3 } $\begin{array}{l}\text { I am in the housing estate opposite } \\
\text { the water tower moving back to } \\
\text { Oasis-Target roundabout }\end{array}$ & $\begin{array}{l}\text { House estate; Watertower } \\
\text { Oasis; Target, Roundabout }\end{array}$ & Allocentric & \\
\cline { 1 - 3 } $\begin{array}{l}\text { I am approx 200 } \mathrm{m} \text { SSE fm coles } \\
\text { h2o tower }\end{array}$ & Coles; Watertower & Extrinsic & duced \\
\hline 4 b. Goto 13th hole & $13^{\text {th }}$ hole [of golfcourse] & Egocentric & \\
\hline
\end{tabular}

\subsection{Mappings Between Internal Representations and the World}

\section{Allocentric FORS Convey Abstract Wayfinding Concepts}

References to landmarks using allocentric (object-centred) FORs appear to reflect awareness of holistic and abstract wayfinding contexts. Wayfinders developed awareness of other's respective locations using imprecise allocentric FORs. These appeared to assist sharing a spatial conceptualization of wayfinding. Wayfinders used landmarks to describe location, rather than direction, in SMSs on their starting locations, for rendezvousing at a known location and during naïve search for the target. In brief initial SMSs (2-6 terms) wayfinders referred to landmarks that they expected others would know (Figs. 2b) (e.g. $\mathcal{A}$ chose the "shopping centre", some distance from his starting location, because it was "a large centralized location with no houses"). SMSs carried relatively low locational specificity (Fig. 3a) but efforts were made to avoid ambiguity (e.g. 4 SMSs were exchanged to clarify that $\mathcal{B}$ was at the bus terminus not a 


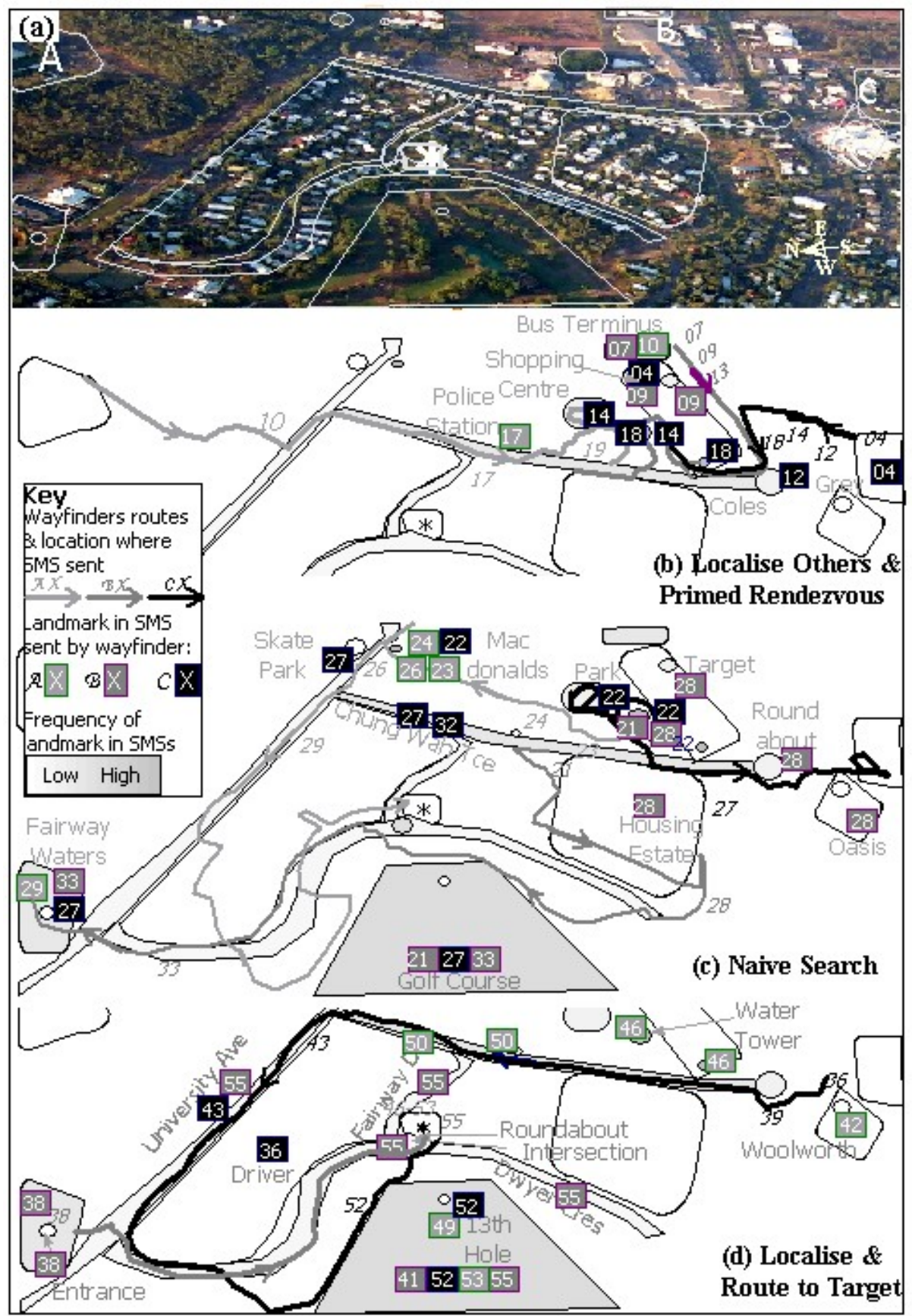

Fig. 2. (a) Aerial view of terrain showing starting locations and example landmarks. Wayfinders' routes and locations where example SMS, referred to in text, were sent during the naturally evolving phases (b, c, d,) of the activity (see key). 
bus stop, Fig. 2b). Imprecise location information was used to co-ordinate rendezvousing at a known place (Figs. 3b, 2b). While no wayfinder was in sight of the water tower, all headed immediately to it without explicating its location or route directions. They said "everyone knows the water satellite tower". Wayfinders used coarse existing knowledge to identify others' locations. When searching for the target (Fig. 2c) they avoided areas others may be, or have been (e.g. "I knew $\mathcal{A}$ was in proximity so moved away from him"). Often wayfinders referred to previous SMSs "to see where [others] said they had been". These expressed imprecise locational information (Fig 3b) but, as $\mathcal{A}$ commented, "visualizing where other people are is easy...".

During the naïve search for the target, wayfinders described and used landmarks with allocentric FORS to spatially relate several districts with course granularity (Figs. 3, 2b). These landmarks often related to semantics about a district. Wayfinders associated the target with public or residential spaces where children might be (e.g. while exploring a housing estate, $\mathcal{B}$ said "single mothers need parks"). Shared allocentric FORs were prone to ambiguity and failed to guide wayfinders' individual searches precisely. When referred to allocentrically, the water tower was misinterpreted twice by different people as a rendezvous rather than a spatial reference. They sought richer descriptions and directional information about headings. Landmarks associated with "nodes" and "districts" seem to contribute to a spatial context for wayfinding that differs from route

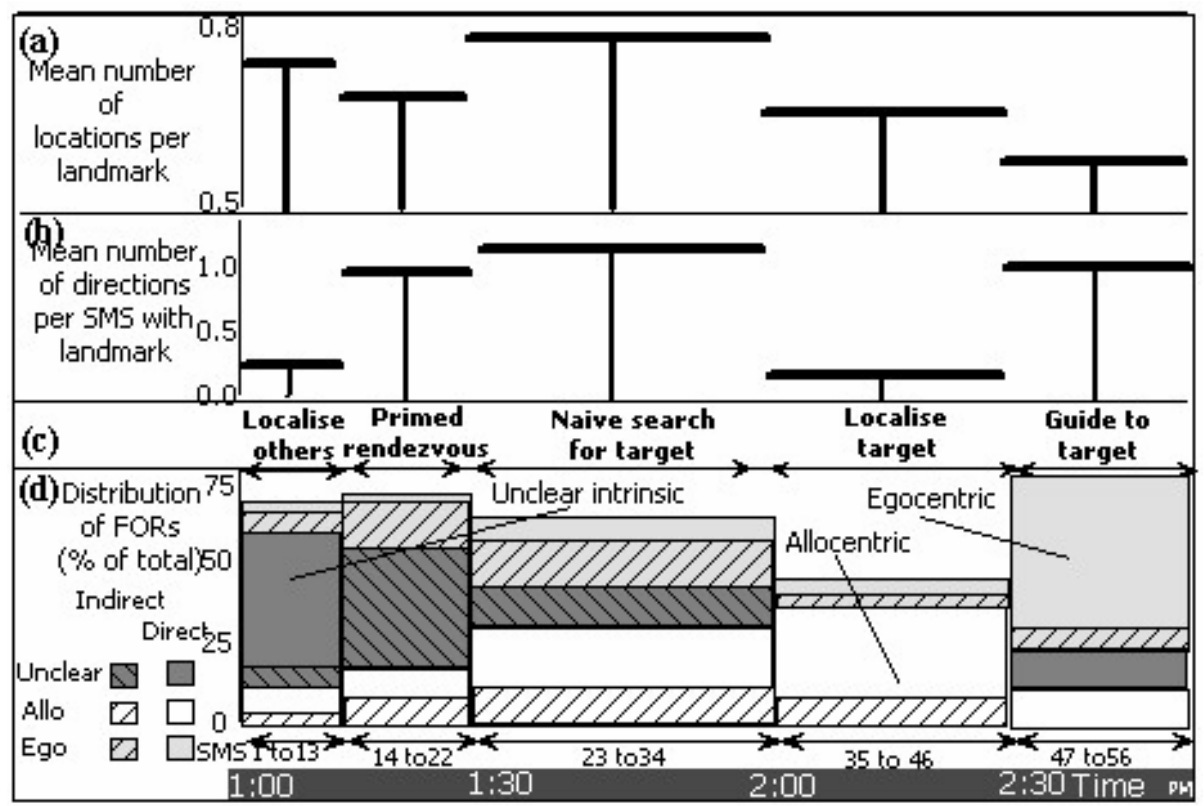

Fig. 3. Characteristics of the use of landmarks in SMS in each (c) phase of wayfinding: (a) Mean number of locations associated with a landmark; (b) Mean number of directions associated with a landmark; (d) Frequency of direct (solid) and indirect (stippled) egocentric, allocentric and non-explicit intrinsic FORs associated with landmarks 
following. Wayfinders primed their search for the target with knowledge limited to places visible from main arterial roads (e.g. McDonalds) or from a hill-top which $\mathcal{A}$ and $C$ used to "see other features" and gain inspiration.

\section{Egocentric FORs Are Associated with Action}

Egocentric (viewer-centred) FORs appeared to relate to movement and direction more directly than allocentric FORs. Wayfinders most accurately and effectively described directional information by describing landmarks with egocentric FORs (Figs. 3b, c). Egocentric structures are thought to more strongly associate with actions than abstract reasoning [28] and correspond more closely with the physical environment than allocentric structures [29]. Conveying a landmark's affordance in verbal route descriptions connects perception to actions [12]. Wayfinders most accurately described and understood route directions when they had direct experience of the landmark relative to that route. This may imply that a landmark's efficacy as a cue en route is relative to subjective perception of it in a route (e.g. $\mathcal{B}$ was able to visualize his heading parallel to a route along the golf course as he had previously walked along the course, Fig. 2d). Generally wayfinders favoured more visual landmarks over road names (e.g. in the CBD's commercial setting, Fig. 2b). However, to describe routes they referred to road names to increase locational specificity (Fig. 2d).

\subsection{Induced FORs Are More Error Prone Than Those Experienced Directly}

Wayfinders induced FORs from limited visual experience of the landmark and/or knowledge of classes of landmarks. An induced FOR is established by performing rotations and relocations of the origin and/or the orientation of an original FOR [30]. Wayfinders tended to induce mentally. On one occasion $\mathcal{A}$ marked a map in the sand to visually assist inducing extrinsic FORs. He described landmarks using, incorrect, compass bearings. Wayfinders described landmarks with similar total numbers of intrinsic FORs but induction and effective use of appropriate FORs increased with familiarity.

Landmarks described by inducing allocentric (object-centred) FORs from limited egocentric (viewer-centred) experience were more error prone and/or confusing than allocentric FORs describing landmarks that were visible to the sender. To increase locational specificity wayfinders combined multiple landmarks, which tended to be on distant arterial roads rather than close to their locales (Figs. 2c, 3a). For example, $\mathcal{B}$ generated allocentric FORs from his limited experience of distal landmarks to report on location and heading. $\mathcal{A}$ arrived at the target by traversing the suburb without scrutinizing interconnecting roads (Fig. 2c) and used allocentric FORs for landmarks denoting routes accessible from $\mathcal{B}$ 's and $C^{\prime}$ 's approximate locations (Fig. 3d). These confused $\mathcal{B}$ and $C$ who were unable to induce the target's location.

Egocentric routes were induced from course allocentric FORs but were error prone without robust knowledge. For example, $\mathcal{A}$ attempted to guide $\mathcal{C}$ along an unknown route between two known landmarks by describing it from an egocentric perspective which he incorrectly inferred from allocentric information. Allocentric knowledge may derive from experience or knowledge of spatial relations holding on a class of landmarks. The golf course offered a set of edges and nodes proximal to the target (Fig. 2d). When asked that the golf course entrance be a reference, $\mathcal{A}$ correctly in- 
duced egocentric perspectives for the route relative to the golf course from allocentric FOR using the landmarks: entrance to, end of and 13th hole. These were not in his sight and he had not seen all of them before. The golf course was the most frequently referenced landmark, used as an initial trajectory and to limit the search extent (Figs. $2 b, c, d)$. This suggests some of its characteristics were functionally significant to the wayfinders. Egocentric perspectives induced from allocentric knowledge of classes of landmarks affected recognition. Wayfinders had difficulty identifying the target as they approached it from an orientation where, as $\mathcal{A}$ said, its "Swings and slides are hidden ... so its park features are obscured" (Fig. 4). A's attention was drawn to the target from $50 \mathrm{~m}$ when he saw a plaque matching the description. $\mathcal{B}$ had previously walked in view without noticing the park; a roundabout obscured his view of the plaque. $C$ was guided to the target from the same direction as $\mathcal{B}$ when $\mathcal{B}$ had missed it.

\section{Landmark Saliency for Memorability: Recalled Landmarks Relate to Decisions}

Differences were observed between landmarks wayfinders used in situ and those they recalled from a route. In the final phase, instructions to guide others to the target, related to edges and paths more than in other phases. $\mathcal{A}$ and $\mathcal{B}$ constructed routes to guide $\mathcal{C}$ by describing landmarks from memory and by checking road names in the vicinity. Yet, when wayfinders retraced their own steps they recognized places but were unaware of road names. $\mathcal{B}$ said "I don't remember road names well [but].. I easily remembered where I had come from". It is proposed that the wayfinders tended to emphasize edges and paths because they were remembered as decision points within the relatively homogeneous suburb. While, this corresponds with saliency when people recall routes; salience for using such directions in situ correlates more strongly with landmarks' appearance than those generated from memory [31].

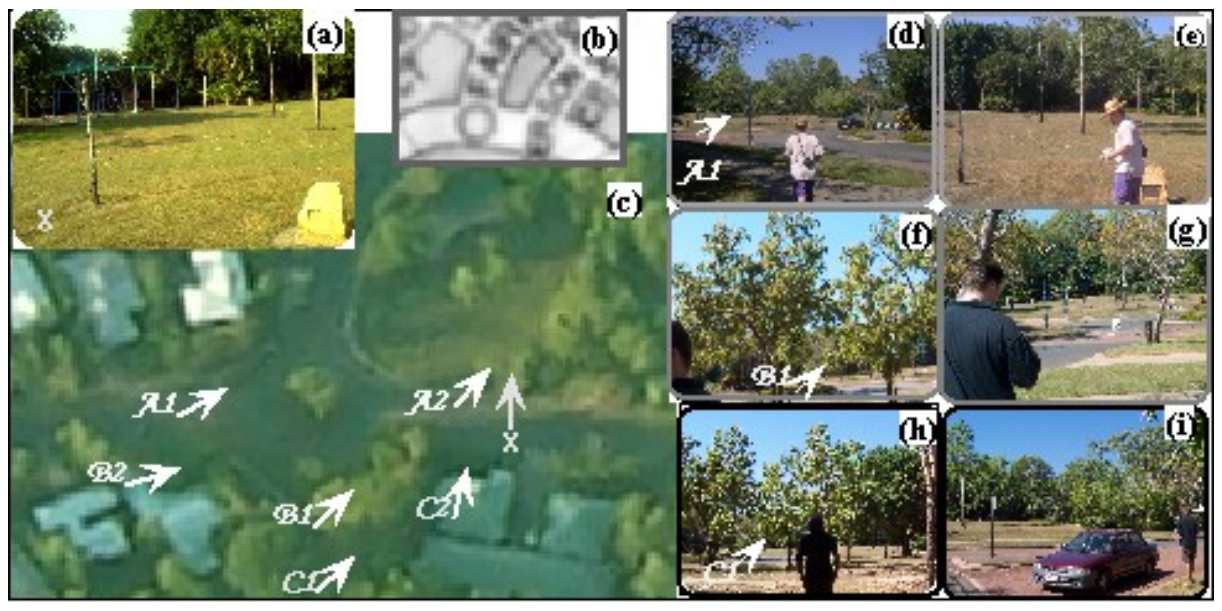

Fig. 4. (a) Park features are visible from limited orientations; (b) Target depicted on a city map; (c) Orientation of wayfinders close to the target; (d, e) $\mathcal{A}$ noticed the plaque from $50 \mathrm{~m}$; (f) $\mathcal{B}$ did not see the target when first in its vicinity; (g) $\mathcal{B}$ and (h, i) $\mathcal{C}$ were guided to the target 


\section{Mappings Between External Representations and the World}

Mappings between a user's internal representations and the world during wayfinding exhibit a dynamic, situated dependency with their environment and subjective wayfinding goals. Such dependencies can be resolved only by gathering data in the specific environments in which people think, reason and act [32]. However, careful analysis of meticulously detailed data which captures user's internal representations created and used in situ can yield generalized patterns to theoretically inform aspects of design.

\subsection{Supporting 'Thrown” Interactions}

Designs enabling accurate direct mapping support "thrown" interactions en route in unfamiliar terrain. We propose that errors in inducing FORs may impede recognizing landmarks in situ from external representations. People will induce some FORs from external representations when they construct internal representations before traversing unfamiliar terrain [e.g. 33]. For example, a person anticipates a landmark's appearance from their intended direction of approach. However, recognising landmarks in situ depends on experience and orientation. People first form egocentric (viewercentred) FORs for an object and these depend on their orientation relative to it during exposure [19]. Tourists are better at recognizing a destination when they view its landmarks from the direction of their first encounter [34] and anecdotes imply that they may not realize they have reached a destination if they are familiar with a single iconic image of it from another angle. Mapping egocentric perspectives delayed identifying features when observers reconstructed wayfinders' routes from images (Fig. 1c) if precise orientation was absent. Users are able to more accurately follow guidance by Global Positioning Systems when it is accompanied by images of proximal landmarks en route [35]. Egocentric representations of objects that can be recognised from any orientation can support users' direct mappings. Users of image-only sequences to follow routes realigned to correspond with an image's orientation and found and recognized features more easily when images contained familiar or predictably shaped objects [36]. Such objects are less easily recalled than unusual objects $[7,37]$.

\subsection{Perspectives and Paradoxes}

We propose that linking route descriptions to coarser graphical depictions of more distal landmarks assists inducing perspectives appropriately. Appropriate induction enables effective "thrown" interactions or more discretionary interactions contextualised by the user's situated and/or global goals. In situ users maintain confidence in route descriptions and their orientation from information between decision points [12] and from distal landmarks and matched landmarks to images which contained multiple features more easily [36]. Coarser depictions of distal landmarks can support accurately inducing FORs to recognise proximal landmarks en route. These should use allocentric FORs to relate proximal to distal landmarks visually and/or semantically. For example depicting prominent, distant landmarks (e.g. mountains, shopping areas) from the perspective of a landmark en route. They should also emphasise "ambiguity of information" [38] if a user's orientation relative to a represented landmark is un- 
predictable. Users following routes using image-only sequences tended to assume that image perspective implied heading and rarely noted triangulating several landmarks [36]. Thus, exposing inconsistency may thwart inducing ineffective FORs.

Emphasising "ambiguity of information" also confers agility in using landmarks for temporally evolving wayfinding goals. Uncertain approximations of landmark position enable sharing abstract wayfinding concepts. For example: by wayfinders to limit the extent of their search and visualise other's trajectories; and, in location-based games, to conceptualise remote player position [21]. Thus, facilitating appropriate induction can support contingent and situated wayfinding [e.g. 11] and enhance communication and decision making during co-located or distributed, collaborative wayfinding [e.g. 21]. A mixed reality game which exposed inconsistencies in locational information engaged player' interpretation of ambiguity [38]. The depictional form of prominent distal landmarks which are allocentrically related and expose inconsistency remains to be explored. We conjecture that caricatures and impressionistic sketches present fertile opportunities for exploring appropriation in situated wayfinding [11]. We propose that exploring such depictions created by users in situ for "naturally" evolving wayfinding goals promises an interesting contribution to research in embodied interactions [5].

\section{Conclusion}

Representations created and used in situ to wayfind can theoretically inform mobile guide design. They identify a need to enable direct mapping to user's perspectives in situ and guide appropriate induction of perspective. Verbal or visual route descriptions from egocentric perspectives should emphasise the salience of proximal landmarks for recognition and action not recall. Co-ordinating these with, macro depictions of distal landmarks will guide perspective and expose ambiguity for situated use.

Acknowledgements. We thank: Darwin Wireless wayfinders for their time and energy; SMSNow for the server; Computer Sciences Corp and Charles Darwin University for support; NC, MP, BP, SV and CL for research assistance and suggestions; $\mathrm{CK}, \mathrm{CG}_{1}$ and $\mathrm{CG}_{2}$, for insightful critique and review; and, $\mathrm{CG}_{1}$ for patience and inspiration.

\section{References}

1. Brown, B., Chalmers, M. Tourism and Mobile Technology. Proc. of the VIIIth European Conference of Computer Supported Cooperative Work Kluwer (2003) 335-354

2. Geldof, S., Dale, R. Improving Route Directions on Mobile Devices. Proc. ISCA workshop on Multi-Modal Dialogue in Mobile Environments Kloster Irsee, Germany (2002)

3. Winograd, T., Flores, F. Understanding Computers and Cognition. Reading, MA, USA: Addison-Wesley Publishing Company (1986)

4. Graham, C., Cheverst, K. Guides, Locals, Chaperones, Buddies and Captains: Managing Trust Through Interaction Paradigms. Workshop on Mobile Guides, Vth Int. Symposium on Human-Computer Interaction with Mobile Devices and Services (2004) 
5. Dourish, P. Seeking a Foundation for Context-Aware Computing. Human-Computer Interaction, (2001).16: 23

6. Siegal, A.W., White, S.H. The development of spatial representations of large-scale environments. Advances in Child Development \& Behavior 10. Academic Press (1975) 10-55.

7. Lynch, K. The Image of the City. MIT-Press, Cambridge, (1960)

8. Thorndyke, P.W. Performance models for spatial and locational cognition. Rand, Washington (1980)

9. Golledge R., Wayfinding Behaviour. John Hopkins UP Baltimore \& London (1999)

10. Taylor, H.A., Tversky, B. Perspectives in Spatial descriptions. J. Memory \& Language (1996)20(5):483-496

11. Brown B., Laurier, E., Designing electronic maps: an ethnographic approach. Map-based mobile services: Theories, Methods and Implementations. Springer-Verlag (2005)

12. May A.E., Ross T., Bayer S.H., Tarkiainen M.J. Pedestrian navigation aids: information requirements and design implications. Pers Ubiquit Comput (2003) 7:331

13. Bidwell, N.J.,Lueg, C Creating a Framework for Situated Way-Finding Research. APCHI New Zealand (2004)

14. Denis, M., Pazzaglia, F., Cornoldi, C., Bertolo, L. Spatial Discourse and Navigation: An Analysis of Route Directions in the City of Venice. App. Cog. Psych. (1999) 13: 145-1

15. Weissensteiner, E., Winter, S. Landmarks in the Communication of Route Directions. GIScience: Lecture Notes in Computer Science, Springer Berlin (2004)

16. Kray, C., Baus J., A Survey of Mobile Guides. Workshop on Mobile Guides, Vth Int. Symposium on Human-Computer Interaction with Mobile Devices and Services (2003)

17. Raubal, M.,Winter, S. Enriching wayfinding instructions with local landmarks. GISscience Lecture Notes in Computer Science Springer, Berlin(2002)

18. Freksa, C., C. Habel C.,Wender K. F. Spatial cognition: An Interdisciplinary Approach to Representing \& Processing Spatial Knowledge, Springer-Verlag, Berlin (1998) 157-175.

19. Iachini, T, Logie R. H. The Role of Perspective in Locating Position in a Real-World, Unfamiliar Environment. Applied Cognitive Psychology (2003) 17:904

20. Goodman, J., Brewster, S. Gray, P. How can we best use landmarks to support older people in navigation Behaviour and Information Technology (2005)

21. Benford, S., Seager, W., Flintham, M., Anastasi R., Rowland D., Humble, J., Stanton D., Bowers, J., Tandavanitj, N., Adams M., Farr, J.R., Oldroyd, A., Sutton J., The Error of Our Ways: The Experience of Self-Reported Position in a Location-Based Game. Proc. Ubiquitous Computing: 6th International Conference, Lecture Notes in Computer Science Springer-Verlag Heidelberg (2004) 3205:70 - 87

22. Grinter, R. E., Eldridge, M. Wan2tlk?: Everyday Text Messaging. Proc. CHI. ACM Press (2003)

23. Ling, R., Birgitte, Y. Hyper-coordination via mobile phones in Norway. In: Perpetual Contact: Mobile Communication, Private Talk, Public Performance Cambridge University Press, Cambridge: (2002) 139-169.

24. Cheverst, K. Fitton, D., Rouncefield, M., Graham, C., 'Smart Mobs' and Technology Probes: Evaluating Texting at Work. Proc. of 11th European Conference on Information Technology Evaluation Royal Netherlands Academy of Arts \& Sciences ( 2004) 11-12

25. Garfinkel, H., Studies in Ethnomethodology, Englewood Cliffs: Prentice-Hall (1967)

26. www.pcc.nt.gov.au/devdem/Statistical\%20Comparisons\%201986\%20to\%202001.pdf

27. SMSNow http://www.nowsms.com

28. Freksa, C. Spatial and temporal structures in cognitive processes. Foundations of Computer Science. Potential Theory Cognition, Springer-Verlag, Berlin (1997) 379-387 
29. Chalmé, S., Visser, W., Denis, M. Cognitive aspects of urban route planning. International Conference on Traffic and Transport Psychology (2000) 145

30. Kray, C Inducing Frames of Reference Workshop on Spatio-Temporal Reasoning at ECAI04, Valencia, Spain (2004)

31. Lovelace, K, Hegarty, M., Montello, D., Elements of Good Route Directions in Familiar \& Unfamiliar Environments. Proc. Int. Conference COSIT, Springer-Verlag (1999) 65-82

32. Suchman, L. Plans and situated actions: the problem of human-machine communication. Cambridge U.P (1987)

33. Michon PE., Denis, M. When and Why are Visual Landmarks Used in Giving Directions? Proc. Int. Conference COSIT, Springer Verlag (2001) 292

34. Allen, G.L., Kirasic, K.C. Effects of the Cognitive Organization of Route Knowledge on Judgments of Macrospatial Distances. Memory \& Cognition (1985) 3: 218-227

35. Schilit, B., LaMarca, A., Borriello, G., Griswold, McDonald, D., Lazowska, E., Balachandran, A., Hong J., Iverson V. Challenge: Ubiquitous Location-Aware Computing and the Place Lab Initiative. First ACM Int. Workshop on Wireless Mobile Applications \& Services on WLAN San Diego, US (2003)

36. Bidwell, N.J., Pictures Made for Walking: Pilots \& Orienteers 1. Proc. Australasian Conference on Computer Human Interaction, Woolongong, Australia (2004)

37. Evans, G. W., Smith, C., Pezdek, K. Cognitive maps and urban form. J. American Planning Associations (1982) 48:232-244

38. Gaver, W.W., Benford, S., Beaver, J., Ambiguity as a resource for design Proc. Conference on Human Factors in Computing Systems ACM Press NY, US (2003) 233 - 240 